\title{
Pulsatile Tinnitus Arising from Aberrant Internal Carotid Artery at Nasopharynx
}

\author{
Chang-Woo Park and Eun-Ju Jeon \\ Department of Otolaryngology, The Catholic University of Korea College of Medicine, Incheon St. Mary's Hospital, Incheon, Korea
}

\author{
Received November 15, 2011 \\ Revised March 25, 2012 \\ Accepted March 29, 2012
}

\section{Address for correspondence \\ Eun-Ju Jeon, MD \\ Department of Otolaryngology, \\ The Catholic University of Korea \\ College of Medicine, \\ Incheon St. Mary's Hospital, \\ 56 Dongsu-ro, Bupyeong-gu, \\ Incheon 403-720, Korea \\ Tel +82-32-280-5887 \\ Fax +82-32-280-5914 \\ E-mail ejmercy@catholic.ac.kr}

Pulsatile tinnitus arising from an aberrant internal carotid artery at the level of the nasopharynx is rarely found. We present a case of a 74-year-old woman complaining of pulsatile tinnitus in the right ear. The tinnitus was not audible by auscultation. Endoscopic examination revealed pulsating swelling of the nasopharyngeal wall on the right side. Computed tomography angiography of the head and neck region showed medial and superficial transposition of the right internal carotid artery at the level of the nasopharynx. The artery was located right next to the orifice of the Eustachian tube and coursed along the distal portion of the tube. The patient's perception of tinnitus was improved after explaining the cause of the tinnitus and reassuring her about thecondition.

Korean J Audiol 2012;16:35-38

\section{Introduction}

The causes of pulsatile tinnitus include arteriovenous malformation or fistula, internal carotid arterial aneurysm or dissection, arteriosclerosis, and an aberrant course of the internal carotid artery, which are the artery-related causes. ${ }^{1)}$ Pulsatile tinnitus arising from an aberrant course of the internal carotid artery is known to be relatively rare and mostly occurs due to an aberrant internal carotid artery in the middle ear. Approximately 95 cases of tinnitus that occur because of an aberrant internal carotid artery in the middle ear have been reported in the worldwide literature thus far and include a report of 2 cases by Kim, et al. ${ }^{2)}$ in Korea. However, 1 case of pulsatile tinnitus due to an extracranial aberrant course of the internal carotid artery reported by Sismanis and Girevendoulis ${ }^{3)}$ in 2008 is the only case of pulsatile tinnitus that has been reported to date.

The authors have experienced a case of pulsatile tinnitus arising from tortuous internal carotid artery which is identified by endoscopic examination as a protruding mass at nasopharyngeal wall. The symptom was improved after explaining and reassuring her status. This paper reports our findings and reviews the relevant literature.

\section{Case Report}

A 74-year-old patient visited our hospital with a chief complaint of pulsatile tinnitus on the right side that began approximately 5 month prior. Symptoms of tinnitus included intermittently hearing a 'toot-toot' sound, which got louder when the surroundings were quiet and when the patient was tired or lying down. There were no otologic symptoms such as otalgia, aural fullness, otorrhea, or hearing loss, etc. The patient was diagnosed with high blood pressure approximately 10 years prior, has been taking medicine continuously, and the blood pressure has been under satisfactory control. Otoscopic examination of the ear showed that the tympanic membranes in both ears were normal, and objective tinnitus was not heard at the time of the head and neck examination. When pressure was applied to the right anterior aspect of the neck or when the neck was rotated to the right and left, tinnitus was not alleviated, and there was no change in the strength of tinnitus even when the Valsalva maneuver was applied. Average air conduction/bone conduction threshold under pure tone audiometry was $35 / 30 \mathrm{~dB}$ HL for the right and 40/30 dB HL for the left, thereby displaying mild descending sensorineural hearing loss on both sides (Fig. 1). The auditory brainstem response test 


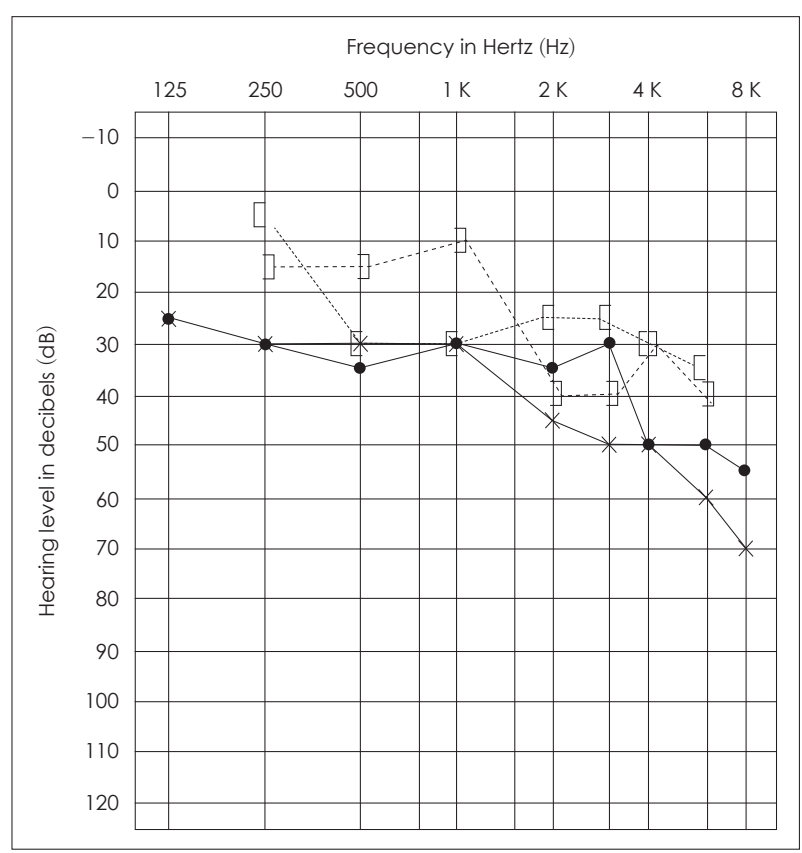

Fig. 1. Pure tone audiogram showing bilateral sensorineural hearing loss of mild degree with descending configuration.
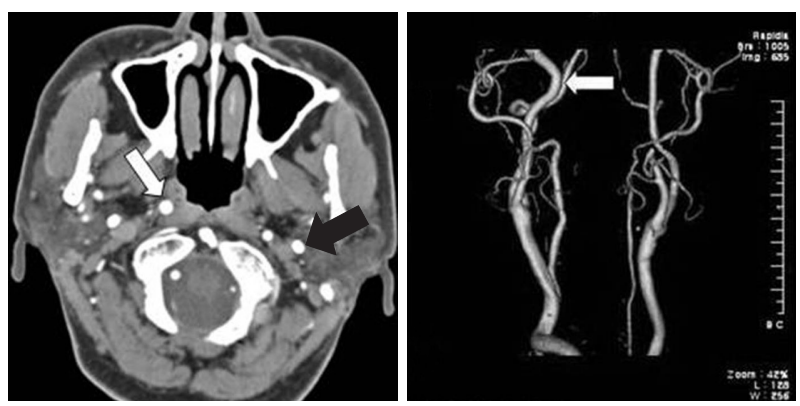

Fig. 2. Head and neck CT angiography shows a medially transposed tortuous internal carotid artery (white arrow) immediately below the skull base at the right side. The artery lies underneath the nasopharyngeal mucosa instead of at its usual site at the parapharyngeal space and runs along the course of the Eustachian tube. The black arrow in the upper image shows the left internal carotid artery with a straight course that runs in the parapharyngeal space. CT: computed tomography.

demonstrated that the threshold was $20 \mathrm{~dB}$ nHL for the right and $40 \mathrm{~dB} \mathrm{nHL}$ for the left, and inter-wave latency and $\mathrm{V}$-wave latency were in their normal range. The transient evoked otoacoustic emission test showed that the right was normal, while the left displayed a drop in response at $3000 \mathrm{~Hz}$. The matching of tinnitus test demonstrated that tinnitus frequency was similar to that of pure tone at $250 \mathrm{~Hz}$, and the loudness of tinnitus displayed a strength of $40 \mathrm{~dB}$ HL. Hematologic and serologic tests revealed normal value. During endoscopic examination of nasopharynx, tortuosity and pulsating of the right external wall of the nasopharynx were observed. With suspicion of a vascular mass in the nasopharynx, head and neck computed tomography (CT) angiography was carried out, and an aberrant course of the right internal carotid artery that protruded towards the nasopharynx and passed in close proximity to the Rosenmuller fossa and the orifice of Eustachian tube was observed during the examination (Fig. 2). In addition, aneurysm of the right side middle cerebral artery and arteriosclerotic calcification and mild occlusion of the internal carotid artery on both sides were observed.

Causes of tinnitus were explained to the patient, and the patient was told to take precautions against damage to the nasopharynx and given a prescription for short-term administration of an anti-anxiety drug. After the causes were explained to the patient, she no longer worried about tinnitus, the symptoms gradually improved, and the patient eventually became unaware of the tinnitus after 2 months.

\section{Discussion}

Pulsatile tinnitus could occur due to causes arising from the artery, vein, and base of skull and temporal bone. Among these, causes related to the artery include arteriovenous malformation or fistula, internal carotid arterial aneurysm, internal carotid artery dissection, arteriosclerosis, and aberrant course of internal carotid artery. ${ }^{1)}$

Tests for identification of the causes of pulsatile tinnitus include microscopic observation of the tympanic membrane, verification of papilledema through fundus examination, observation of movement of the tympanic membrane during breathing, observation of the orifice of the Eustachian tube using transnasal endoscopy, observation of loss of tinnitus following application of pressure to the neck, CT angiography, verification of superior semicircular canal dehiscence under high resolution temporal bone $\mathrm{CT}$, carotid artery ultrasonography, detection of benign intracranial hypertension through magnetic resonance imaging, magnetic resonance angiography, magnetic resonance venography, and examination of the fundus, etc. ${ }^{1)}$

The type of aberrant course of the internal carotid artery that can induce pulsatile tinnitus is the aberrant course of the internal carotid artery in the middle ear, which is thought to be the result of failure to generate the bony plate that should exist between the carotid artery and tympanic cavity due to an embryogenic blood vessel that continues to exist by being connected to the middle ear during its formation in the embryogenic stage. Such dehiscence of the internal carotid artery canal in the middle ear is thought to exist in approximately $1 \%$ of the world's population. ${ }^{4)}$

Another aberrant course of the internal carotid artery can occur at the extracranial neck level. According to most anatomy textbooks, after the internal carotid artery of the neck bran- 
ches from the common carotid artery at the hyoid bone level, it enters the middle cranial fossa through the foramen lacerum by being wrapped by the carotid sheath and moving upwards vertically, from the parapharyngeal space to the base of the skull. ${ }^{5)}$ However, there have been reports of an aberrant course of the internal carotid artery in which the internal carotid artery in the neck courses in close proximity to the pharyngeal wall by departing from the parapharyngeal space and displaying abnormal appearances, including tortuosity from the straight line course, kinking, or coiling. The prevalence rate of such a manifestation has been reported to be in the range of $10-40 \%$ in studies using anatomy, ultrasonography, and angiography. ${ }^{6,7)}$ Closer examination of these reports include the report by Macchi, et al. ${ }^{6}$ that it was possible to discover internal carotid artery deformation (tortuosities, kinks, or coiling) in $26.5 \%$ of healthy people with no symptoms using carotid artery Doppler ultrasonography that was carried out in 100 healthy adults as the subjects. On the other hand, Pancera, et al.," in analysis of carotid artery Doppler ultrasonic imaging in 590 subjects, discovered appearance of kinking of the carotid artery in $31.2 \%$ of subjects and thought that increase in the internal pressure of the artery acts as the cause for inducement of deformation of the appearance of the internal carotid artery, since the prevalence rate was significantly higher in a group with high blood pressure.

Causes of an aberrant course of the internal carotid artery are thought to be largely due to congenital developmental abnormality and changes according to age. The internal carotid artery is derived from the 3rd brachial cleft artery and the cranial level of the dorsal aorta in the embryonic period; in the 8th week of embryogenesis, the internal carotid artery uncoils as the dorsal aortic root descends into the chest. Failure of this process, incomplete development, or excessive growth of the internal carotid artery can induce an aberrant course of the internal carotid artery that includes tortuosity and coiling. The fact that an aberrant course of the internal carotid artery is discovered in $5-10 \%$ of subjects during infancy provides evidence that supports the theory of embryological developmental disturbance. ${ }^{8)}$

On the other hand, as age increases, the prevalence rate of an aberrant course of the internal carotid artery of the neck increases. Occurrences of arteriosclerosis, high blood pressure, lowering of elasticity of blood vessels, and fibromuscular dysplasia, which occur in accordance with an increase in age, are thought to act as factors that induce an aberrant course of the internal carotid artery. ${ }^{9)}$

As such, an aberrant course of the internal carotid artery of the neck can cause cerebrovascular insufficiency in 4-16\% of cases and can induce cerebrovascular embolism, headache, and intermittent stenosis of the carotid artery. ${ }^{10)}$ Otorhinolaryngologically, dysphagia, foreign body sensation in the neck, pain in the neck, dizziness, and tinnitus could be induced. ${ }^{11)}$

Three cases of pulsatile tinnitus due to an aberrant course of the internal carotid artery of the neck have been reported for the first time by Sismanis and Girevendoulis ${ }^{3)}$ in 2008. In 2 of the cases, it was possible to auscultate objective pulsatile tinnitus at each external auditory canal and infra-auricular region, and tortuosity of the internal carotid artery was observed below the cranium base in all 3 patients, with 1 case accompanied by coiling the of internal carotid artery. No special treatment was given to all 3 patients and, in 1 of the patients, the symptoms naturally disappeared 10 months after the examination. Regarding the phenomenon of natural disappearance of symptoms, Sismanis and Girevendoulis ${ }^{3)}$ presumed that tinnitus stopped due to disappearance of turbulence in the blood flow as the internal carotid artery continued to have changes.

In our case, it was possible to observe appearances of tortuosity and pulsation of the nasopharyngeal mucous membrane at the immediately posterior aspect of the orifice of the Eustachian tube due to an internal carotid artery that had been transpositioned internally. It is thought that the pulsatile tinnitus that the patient complained of was generated due to conveyance of pulsating sound created by the flow of blood in the internal carotid artery with tortuosity to the middle ear cavity through the Eustachian tube.

In our case, although the patient also had high blood pressure, arteriosclerosis, and aneurysm, which are considered to be causes of pulsatile tinnitus, it is thought that these would not have been the direct cause of the pulsatile tinnitus on the right side. If high blood pressure, which is a systemic disease, and the arteriosclerosis present in the internal carotid artery on both sides acted as the causes of tinnitus, then the pulsatile tinnitus would have been manifested on both sides. However, since high blood pressure and arteriosclerosis are considered to be the causes that induce an aberrant course of the internal carotid artery (tortuosity, kinking, and coiling) by causing hemodynamic changes, ${ }^{7,9)}$ they are thought in our case to have had an indirect influence by inducing tortuosity of the nasopharynx section of the internal carotid artery on the right side.

Aneurysm of the internal carotid artery or vertebral artery is known to be one of the rare causes of pulsatile tinnitus, with aneurysm of the internal carotid artery of the petrous ${ }^{13)}$ and aneurysm of the sigmoid sinus ${ }^{14)}$ being reported as the main causes of pulsatile tinnitus. In contrast, pulsatile tinnitus due to aneurysm of the cerebral artery other than a dissecting aneurysm is highly rare. ${ }^{15)}$ Although the aneurysm that was present in our case was on the same side as the location of tinnitus, it was a relatively small aneurysm that occurred in the middle 
cerebral artery in an area irrelevant to the internal auditory canal and petrous and was not a dissecting aneurysm. Therefore, the possibility of pulsatile tinnitus due to aneurysm was excluded.

In our case, pulsatile swelling that accompanied pulsatile tinnitus was observed under endoscopic examination that was carried out at the time of the patient's visit to the hospital. Head and neck CT angiography was selected for further examination under the suspicion of a vascular mass and demonstrated an aberrant course of the internal carotid artery.

Although surgical treatment, such as reconstruction of the internal carotid artery or endarterectomy, is necessary for the treatment of pulsatile tinnitus due to an aberrant course of the internal carotid artery if symptoms of apoplexy, transient ischemic attack of the cerebrum, destruction of surrounding structures, occurrence of aneurysm, and cerebrovascular embolism, are present, it would be advisable to limit surgical treatment to patients with serious cranial nerve symptoms, since manipulation of the internal carotid artery can elicit detrimental hemorrhage, apoplexy, or transient ischemic attack. ${ }^{12)}$ When patients' concerns about tinnitus are addressed by explaining its causes, pulsatile tinnitus disappears in many cases, ${ }^{1)}$ or, even if tinnitus is sustained, the patient must not become fearful of or worry about the tinnitus itself. In addition, patients must be guided to avoid situations that can induce future damage to the internal carotid artery by providing them with information on the disease. For the patient in our case, tinnitus disappeared without particular surgical treatment, because the patient's concerns about tinnitus were relieved after the clinician provided the explanation that tinnitus is not a special disease, but rather, it is manifested due to alteration in the course of the carotid artery and prescribed an anti-anxiety drug. As another mechanism for the natural disappearance of tinnitus, it is thought that there is a possibility that noise in blood flow and pulsatile tinnitus disappeared due to gradual reduction in turbulence in the blood vessel that formed at the initial stage as alteration of the internal carotid artery continued to proceed.

\section{REFERENCES}

1) Mattox DE, Hudgins P. Algorithm for evaluation of pulsatile tinnitus. Acta Otolaryngol 2008;128:427-31.

2) Kim JH, Kim DI, Kim HJ, Yoon DY. Aberrant internal carotid artery in the middle ear. Korean J Otolaryngol-Head Neck Surg 1997; 40:619-23.

3) Sismanis A, Girevendoulis A. Pulsatile tinnitus associated with internal carotid artery morphologic abnormalities. Otol Neurotol 2008; 29:1032-6.

4) Botma M, Kell RA, Bhattacharya J, Crowther JA. Aberrant internal carotid artery in the middle-ear space. J Laryngol Otol 2000;114:784-7.

5) Gray H. Cardiovascular: internal carotid artery. In: Williams PL, Bannister LH, Berry MM, et al., 38th ed. Gray's Anatomy, Edinburgh, UK: Churchill Livingstone, 1995;1523-9.

6) Macchi C, Gulisano M, Giannelli F, Catini C, Pratesi C, Pacini P. Kinking of the human internal carotid artery: a statistical study in 100 healthy subjects by echocolor Doppler. J Cardiovasc Surg (Torino) 1997;38:629-37.

7) Pancera P, Ribul M, Presciuttini B, Lechi A. Prevalence of carotid artery kinking in 590 consecutive subjects evaluated by Echocolordoppler. Is there a correlation with arterial hypertension? J Intern Med 2000;248:7-12.

8) Ricciardelli E, Hillel AD, Schwartz AN. Aberrant carotid artery. Presentation in the near midline pharynx. Arch Otolaryngol Head Neck Surg 1989;115:519-22.

9) Del Corso L, Moruzzo D, Conte B, Agelli M, Romanelli AM, Pastine F, et al. Tortuosity, kinking, and coiling of the carotid artery: expression of atherosclerosis or aging? Angiology 1998;49:361-71.

10) Morgenlander JC, Goldstein LB. Recurrent transient ischemic attacks and stroke in association with an internal carotid artery web. Stroke 1991;22:94-8.

11) Iwasaki S, Fujishiro Y, Abbey K. Glossopharyngeal neuralgia associated with aberrant internal carotid artery in the oropharynx. Ann Otol Rhinol Laryngol 2002;111:193-5.

12) Illuminati G, Ricco JB, Caliò FG, D’Urso A, Ceccanei G, Vietri F. Results in a consecutive series of 83 surgical corrections of symptomatic stenotic kinking of the internal carotid artery. Surgery 2008; 143:134-9.

13) Palacios E, Gómez J, Alvernia JE, Jacob C. Aneurysm of the petrous portion of the internal carotid artery at the foramen lacerum: anatomic, imaging, and otologic findings. Ear Nose Throat J 2010;89: 303-5.

14) Gologorsky Y, Meyer SA, Post AF, Winn HR, Patel AB, Bederson JB. Novel surgical treatment of a transverse-sigmoid sinus aneurysm presenting as pulsatile tinnitus: technical case report. Neurosurgery 2009;64:E393-4; discussion E394.

15) Sismanis A. Pulsatile tinnitus. Otolaryngol Clin North Am 2003; 36:389-402, viii. 